\title{
Fernando Cassinello Pérez, arquitecto poliédrico
}

\section{Fernando Cassinello Pérez, polyhedral architect}

\author{
M. Centellas Soler ${ }^{(*)}$, J. F. García-Sánchez ${ }^{(* *)}$
}

\section{RESUMEN}

La obra del arquitecto almeriense Fernando Cassinello Pérez (Almería, 1928-Madrid, 1975) se apoya sobre tres pilares: la producción arquitectónica centrada en el proyecto y construcción de viviendas y establecimientos hoteleros; la actividad académica como Catedrático de Construcción en la Escuela de Arquitectura de Madrid, y la inquietud investigadora desarrollada en el Instituto Eduardo Torroja del que llegó a ser director, y ejercida como autor de libros y artículos de investigación, como conferenciante divulgador, como ponente en importantes congresos o como miembro activo de asociaciones internacionales vinculadas a la construcción con hormigón. Este artículo hará un repaso de su prolífica actividad como arquitecto poliédrico y dará cuenta de las interesantes obras que proyectó y construyó apoyándose en una dilatada cultura arquitectónica contemporánea e internacional.

Palabras clave: Cassinello; arquitecto; siglo xx; estructura; hormigón; vivienda; investigación; Almería.

\section{ABSTRACT}

The work of architect Fernando Cassinello Pérez (Almería, 1928-Madrid, 1975) rests on three pillars: architectural production focused on the design and construction of housing and hotel establishments, academic activity as Professor of Construction at the Escuela de Arquitectura de Madrid, and research concern developed in the IETcc of which he became director, and exercised as an author of books and research papers, as disseminator lecturer, speaker at major conferences or as an active member of international associations linked to construction with concrete. This article will review his prolific activity as polyhedral architect and will find interesting works that designed and built by relying on extensive contemporary and international architectural culture.

Keywords: Cassinello; architect; 2oth Century; structure; concrete; housing; research; Almería.

(*) Universidad Politécnica de Cartagena (Cartagena, Murcia, España).

(**) Universidad Politécnica de Madrid (Madrid, España).

Persona de contacto/Corresponding author: miguel.centellas@upct.es (M. Centellas Soler); jf.gs@upm.es (J. F. García-Sánchez). ORCID: http://orcid.org/oooo-0002-7264-7044 (M. Centellas Soler); http://orcid.org/oooo-0oo1-7509-9752 (J. F. García-Sánchez)

Cómo citar este artículo/Citation: Centellas Soler, M., García-Sánchez, J. F. (2016). Fernando Cassinello Pérez, arquitecto poliédrico. Informes de la Construcción, 69(545): e174, doi: http://dx.doi.org/10.3989/ic.16.083.

Copyright: (C) 2017 CSIC. Licencia / License: Salvo indicación contraria, todos los contenidos de la edición electrónica de Informes de la Construcción se distribuyen bajo una licencia de uso y distribución Creative Commons Attribution License (CC BY) Spain 3.o. 
El arquitecto Fernando Cassinello desarrolló su actividad profesional principalmente en Almería y es en esta provincia donde construyó la mayoría de sus edificios. La fortuna sonríe a los audaces, pero también a los trabajadores: sólo en los archivos del Colegio de Arquitectos de Almería se han localizado alrededor de 500 registros de obras llevadas a cabo en poco más de 21 años, los que van desde la obtención del título de arquitecto en 1954 hasta su prematuro fallecimiento en 1975. Si en Almería el arquitecto por excelencia de la primera mitad del siglo $\mathrm{xx}$ fue Guillermo Langle Rubio - que ha sido homenajeado con algunas exposiciones y libros- la segunda mitad de ese siglo es la época de Fernando Cassinello a quien se le debe el reconocimiento necesario ${ }^{1}$ y este texto pretende contribuir al estudio de su obra y de su actividad docente e investigadora.

Nacido en Almería el 30 de marzo de 1928, fue el segundo hijo de una familia de cinco hermanos. Estudió la enseñanza secundaria en el Colegio de La Salle, después se trasladó al Colegio de Huérfanos de Valladolid y posteriormente en 1945 realizó el curso introductorio en la Universidad de Madrid, ingresando en la Escuela de Arquitectura al año siguiente, y titulándose el 28 de julio de 1954. Durante los tres últimos años de su formación disfrutó de la Beca «Carmen del Río» de la Real Academia de Bellas Artes de San Fernando (1). Obtuvo el título de Doctor arquitecto el 12 de enero de 1961. En 1952 se casó con María Josefa Plaza García y fruto de ese matrimonio nacieron cinco hijos, de los cuales dos, Pepa y Adela, han continuado con la profesión del padre (Figura 1).

$\mathrm{Su}$ actividad profesional descansa sobre tres pilares: la docencia, la investigación y la construcción, viviendo a caballo entre Madrid y Almería, donde tuvo sendos estudios profesionales ${ }^{2}$. Aunque también construyó en otras ciudades españolas: Pamplona, Murcia, Alcalá de Guadaira (Sevilla), etc. Fue profesor en la Escuela de Arquitectura de Madrid e investigador del IETcc, además dirigió la revista Informes de la Construcción a finales de los años cincuenta, publicación donde principalmente se difundió su obra ${ }^{3}$.

De carácter expandido y centrífugo, muchas veces se situó en el límite de sus propias posibilidades ${ }^{4}$. En un viaje a Nigeria, por motivos profesionales, contrajo una enfermedad tropical que acabaría finalmente con su vida en Madrid, el 19 de agosto de 1975 .

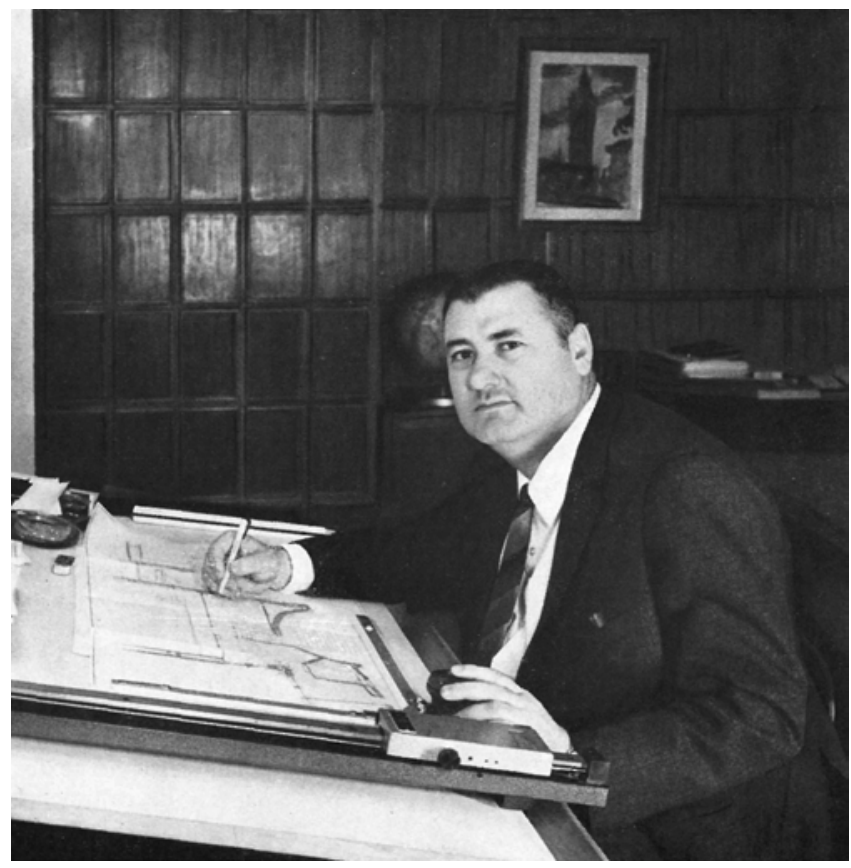

Figura 1. Retrato de Fernando Cassinello. [Fuente: Revista Informes de la Costrucción, IETcc, CSIC].

\section{EL PROFESOR Y ARTICULISTA}

$\mathrm{Su}$ vocación docente fue prematura: inmediatamente a la conclusión de sus estudios ingresó durante el curso 19541955 en la Escuela de Arquitectura de Madrid como profesor ayudante de «Construcción I y II» ${ }^{5}$. Durante los siguientes cursos impartió docencia en el área de Construcción, Composición y Expresión gráfica ${ }^{6}$. Y el 21 de junio de 1967 consigue la plaza de Catedrático numerario del grupo XVII de la Escuela de Arquitectura de Madrid, impartiendo docencia en «Construcción II» y «Cimentaciones especiales» (Plan 1957) y «Construcción IV» (Plan 1964) (Figura 2). Tal y como comenta Ricardo Aroca: «Era imposible no quererlo, pese a ser un triunfador, catedrático de Construcción al primer intento, después de una sorprendente tentativa de serlo de iTeoría del Arte! en competencia con Víctor D’Ors y a la vez una carrera meteórica en el Torroja, donde era Director en funciones mientras proyectaba y dirigía importantes edificios» (2).

\footnotetext{
Lamentablemente Fernando Cassinello es injustamente recordado por el suceso del edificio Azorín. Las hemerotecas y parte de la memoria colectiva insisten en vincularlo con ello y este artículo pretende destacar su labor profesional, académica e investigadora y ponerla en valor como uno de los arquitectos almerienses más importante de la segunda mitad del siglo xx.

2 Tuvo dos estudios profesionales: en Madrid, en la calle Apolonio Morales, y en Almería, el primero en la calle Rueda López y, después de proyectar y construir el edificio Belén, en la confluencia de la Rambla con la avenida de la Estación, se trasladó a la primera planta del mismo. 3 Después de su fallecimiento se ha publicado escasa información sobre ella, pudiendo destacar algunos edificios publicados en: Almería. Guía de arquitectura y en La arquitectura del sol_Sunland architecture. Se publicaron el Gran Hotel Almería, la Capilla de Nuestra Señora de los Vientos (Roquetas de Mar) y los apartamentos El Palmeral (Aguadulce). Villanueva Pleguezuelo, E. (2003). Desengaño veraniego. En VV. AA. La arquitectura del sol Sunland Architecture. Barcelona: Colegios de Arquitectos de: Cataluña, Comunidad Valenciana, Islas Baleares, Murcia, Almería, Granada, Málaga y Canarias.

${ }^{4}$ Como indicaba su hermana María: «Todo lo malo que le pasó en vida fue por un exceso de confianza.» Conversación mantenida con su hermana María Cassinello en su casa de Almería el 4 de noviembre de 2013.

5 En su CV personal de 1969 aparece que, durante este curso 1954-1955, impartió la asignatura de «Construcción I y II». Pero en la hoja de servicios de la Escuela de Arquitectura de Madrid certifica que fue Profesor ayudante interino de «Geometría Descriptiva» y «Perspectiva y sombras».

${ }^{6}$ De 1955-1958 fue profesor auxiliar de «Geometría descriptiva. Perspectivas y sombras». De 1958-1960 fue el profesor encargado de la cátedra de «Materiales de construcción». Durante el curso 1961-1962 compaginó la docencia como profesor auxiliar en «Construcción I» y profesor adjunto en el plan moderno de «Composición II». Durante el curso 1962-1963 ya es el profesor encargado de la cátedra de «Construcción I». Durante los cuatro cursos del 1963-1967 es el profesor encargado de las cátedras de «Construcción arquitectónica y máquinas y medios auxiliares». Y, paralelamente, en los años 1963, 1966 y 1968 fue profesor jefe del grupo de Composición de los Cursos de Estudios Mayores de la Construcción (CEMCO) para Postgraduados Hispano-Americanos en Arquitectura e Ingeniería.
} 

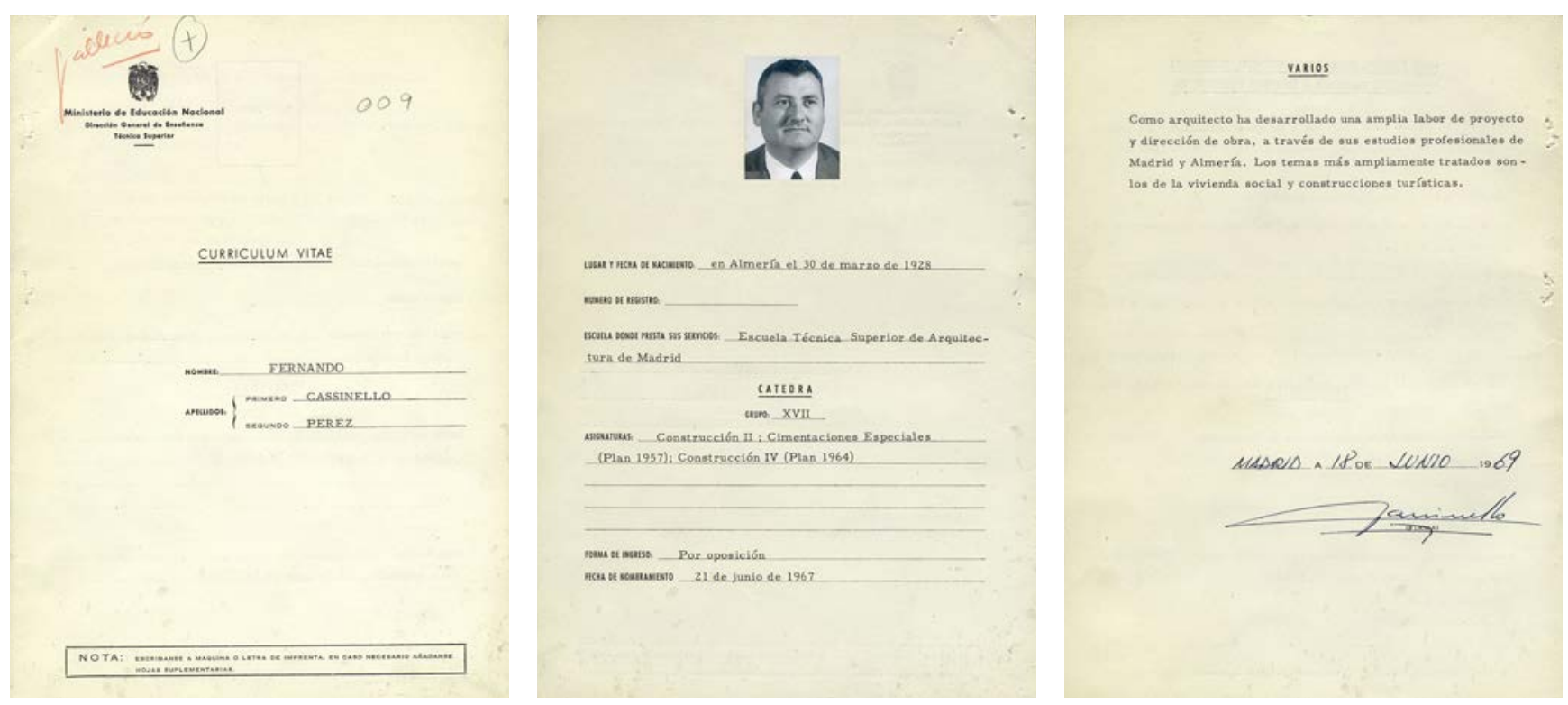

Figura 2. Curriculum Vitae de Fernando Cassinello Pérez (18 de junio de 1969) [Fuente: AGA, Archivo General de la Administración].

En 1950 empezó a colaborar como traductor de alemán en Informes de la Construcción donde escribió alrededor de 120 artículos (3). El primero apareció en 1952 siendo todavía estudiante, ya que aún le faltaban dos años para titularse como arquitecto, y estaba firmado con las iniciales F. C. P. (4) y así los siguió suscribiendo hasta finales de 1955. En su mayoría se trataba de artículos extraídos de revistas extranjeras relativos casi siempre a edificios situados en Estados Unidos y Europa, con escasa aportación personal. El primer texto firmado con el primer apellido es «Arquitectura escolar» en el número 74 de octubre de 1955 (5). A partir de esa fecha aparecen textos más técnicos en relación al hormigón y las estructuras, y también algunos sobre sus obras, como el artículo dedicado a la reforma para un local comercial en Almería (1955) (6) - una zapatería para la familia de su mujer Pepa Plaza - o el dedicado a la Capilla en Costillares (1957) (7). Esta obra también la publicó en la Revista Nacional de Arquitectura (8). O el artículo sobre la figura de Eduardo Torroja (1961) (9), publicado en el número 47 de la revista Cuadernos de Arquitectura y Urbanismo, editada en Barcelona y donde también se publicaron dos de sus obras: El Palmeral (1968) (10) y Los Tritones (1967) (11). Recibió un premio por su artículo «Influencia del hormigón en la estética de la construcción», publicado en el número 310 de la revista Cemento y hormigón (1961). Autor prolífico, escribió además tantos otros en distintas revistas como Hormigón y Acero, Revista Nacional de Arquitectura, Revista de Vivienda y Urbanismo, Boletín de la Sociedad Española de Cerámica, Hogar y Arquitectura, la revista Afal, Boletín de la Asociación Española de Hormigón Pretensado, Revista Materiales, Maquinaria y métodos para la construcción, Cuadernos hispanoamericanos, etc.

Enamorado del ladrillo, escribió varias monografías, muchas de ellas publicadas por el IETcc: El ladrillo y sus fábricas (1958), Arcos de ladrillo (1958), Los amigos del ladrillo (1960), Bóvedas y cúpulas de ladrillo (1964), Muros de carga de fábricas de ladrillo (1964). Además, por sus amplios conocimientos sobre este material y sus fábricas, en 1965 fue llamado a formar parte de la Comisión creada en el Ministerio de la Vivienda ${ }^{7}$ para la redacción de una de las Normas más importantes de la época, la MV 201 de 1972 sobre «Muros resistentes de fábrica de ladrillo». Entre los textos sobre estructuras merecen citarse Bruselas, alarde estructural (1959) y El arquitecto y la estructura (1970).

En 1973 se inicia por la Editorial Rueda la serie Construcción, con un primer volumen sobre Carpintería dedicado a la madera, seguido de Hormigonería (1974) que, sin lugar a dudas, es su publicación más conocida y divulgada ${ }^{8}$. De forma clara y concisa explica los componentes y la ejecución de ese material documentado, en muchos casos, con sus propias obras. Cuando falleció estaba preparando un tercer volumen dedicado a la piedra y que se iba a titular Cantería.

\section{EL INVESTIGADOR Y DIVULGADOR}

Según Miguel Fisac, le debe a Fernando Cassinello su interés por las estructuras, después de una conversación: «Me di al final cuenta de esos olvidos y deficiencias y un cariñoso reproche, en 1958, de mi amigo y compañero Fernando Cassinello, sobre mi despreocupación por la investigación estructural de mis proyectos, me hizo reconsiderar la cuestión» (12).

Fernando Cassinello tuvo desde su posición de inicial colaborador en Informes de la Construcción una relación muy directa con el ITcc, Instituto Técnico de la Construcción y el Cemento (IETcc, Instituto de Ciencias de la Construcción Eduardo Torroja, a partir de 1961) del Patronato «Juan de la Cierva» del CSIC. En el IETcc fue Jefe del Departamento de Construcción y Director accidental del Instituto desde 1968 hasta 1970, debido a que el director titular, Jaime Nadal, fue nombrado Director General de Obras Hidráulicas del Ministerio de Obras Públicas (13). Como investigador Jefe del

\footnotetext{
7 Junto con los arquitectos Juan del Corro, Luis Felipe Rodríguez, Javier Lahuerta, Francisco Bassó y Joaquín Fernández.

8 Construcción Hormigonería era un libro de texto casi obligatorio para todos los estudiantes de la época en la Escuela de Madrid, y que todavía hoy se sigue recomendando en muchas guías docentes de asignaturas de construcción.
} 
Departamento de Construcción del IETcc dirigió los Proyectos de Investigación que se desarrollaban en dicho Departamento: bien a corto plazo, como consecuencia de peticiones de expedientes para la industria, así como a largo plazo, como tema de trabajo del propio Centro, y cuyos resultados fueron recogidos en las publicaciones que se han señalado anteriormente. Junto con el ingeniero Carlos Barredo construyó, a mediados de los años sesenta, la Casa Barredo en San Fernando de Henares, con una terraza en voladizo de $15 \mathrm{~m}$ construida mediante dovelas de hormigón postesado y que fue récord mundial en su momento (Figura 3).
Su interés por el hormigón le llevó a ser Director de la Asociación Española del Hormigón Pretensado (AEHP) 9 . Y Presidente de la Asociación Técnica Española del Pretensado (ATEP). Fue Full Member de la International Association for Shell Structures (IASS) fundada en 1958 por Eduardo Torroja. Cassinello también fue editor del Boletín que esa asociación de estructuras laminares editaba.

En 1957 formó parte de la Comisión Nacional de Productividad Industrial que viajó a Estados Unidos para establecer contactos con entidades americanas. Ese viaje le sirvió para
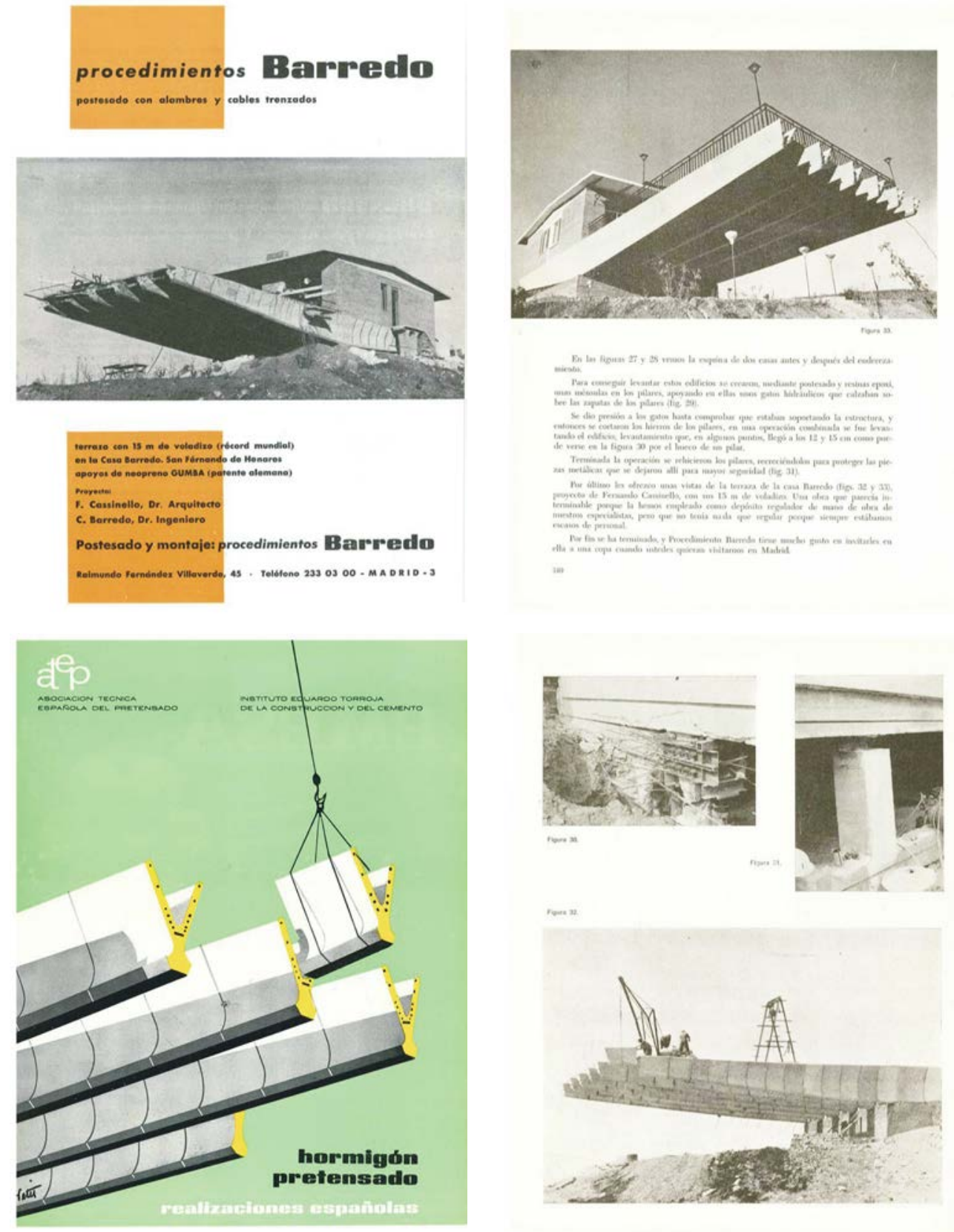

Figura 3. Anuncio de la empresa Barredo donde se ve la Casa Barredo en construcción (Hormigón y Acero, 80), e imágenes incluidas en un artículo de la revista (Hormigón y Acero, 94) [Fuente: Revista Hormigón y Acero, IETcc, CSIC].

\footnotetext{
9 «En el año 1949 se crea la AEHP, Asociación Española de Hormigón Pretensado, adscrita al ITcc, Instituto Técnico de la Construcción y el Cemento, como activo medio de información y difusión. Y cuando posteriormente, en el año 1951, se funda la FIP, Federación Internacional de Pretensado, es la AEHP, la llamada a representar a España, no independizándose del Instituto hasta la década de los setenta. Calavera-Ruiz, J., Cassinello, P., Fernández-Alba, A., et al. (2008). El espíritu impreso de una idea (p. 23). Madrid: Instituto de Ciencias de la Construcción Eduardo Torroja, CSIC.
} 
visitar en Taliesin West a Frank Lloyd Wright (1867-1959), con el que entablaría una buena amistad. También se relaciona con otro maestro internacional, Richard Neutra (1892-1970) ya que éste fue el máximo colaborador extranjero de la revista Informes de la Construcción durante once años (1956-1967), escribiendo artículos personalmente o facilitando el trabajo para que fueran publicados. En 1968, el IETcc publicó el libro R. J. Neutra, con prólogo y edición de Fernando Cassinello, en el cual se recogen no sólo una gran parte de los más de 30 artículos escritos por Neutra, sino también otros escritos inéditos legados por el propio Richard Neutra y su esposa Dione (14). En una nota necrológica - brillantemente redactada- publicada en Informes de la Construcción (1965) (15), Cassinello confiesa que conoció a Le Corbusier en una entrevista que le realizó en su estudio parisino de la rue de Sèvres.

Sus primeras ponencias a finales de 1957 en la Escuela de Madrid trataron la obra de Mies van der Rohe, Richard Neutra, Frank Lloyd Wright, Le Corbusier o Antonio Gaudí. Posteriormente la mayoría de sus conferencias estuvieron relacionadas con cuestiones técnicas sobre el ladrillo, el hormigón armado, la industrialización y las estructuras, principalmente las pretensadas y laminares. Pero también sobre la vivienda social o las construcciones hoteleras.

Asistió, junto a Javier Lahuerta, en Milán al Congreso Internacional organizado por RILEM en 1962 presentando la ponencia «Resistence de Murs et Cloisons de briques». Y a las reuniones en París (1962), Londres (1963), Oslo (1965) y Kiev (1967) de las comisiones W-23 (Wall Structures) del CIB, Conseil International du Bâtiment ${ }^{10}$.

En el verano de 1963 realizó un viaje a Suramérica y fue ponente sobre temas estructurales en Río de Janeiro, Montevideo, Buenos Aires, Santiago de Chile y Bogotá.

En 1974 lee la ponencia «El hormigón pretensado en la edificación española», como delegado del grupo español, durante el VII Congreso Internacional de la Federación Internacional de Pretensado (FIP), celebrado en Nueva York durante los días 25 a 31 de mayo de 1974.

Fue un asiduo conferenciante ${ }^{11}$ y en el período comprendido entre 1957 y 1969 impartió alrededor de unas setenta charlas, la mayoría en aquella España y con aquellas comunicaciones ${ }^{12}$.

\section{EL ARQUITECTO Y CONSTRUCTOR}

En 1954, cuando terminó la carrera, Almería se encontraba en el vagón de cola de España en lo que a progreso económico se refiere. Los dos motores de la economía almeriense de la segunda mitad del siglo xx, la agricultura y el turismo, todavía no se habían desarrollado. El Instituto Nacional de Colonización construyó los primeros pueblos en la provincia en ese año (la ampliación de Roquetas de Mar y El Parador) (16) y hubo que esperar hasta 1964 para que se concediera en España el primer Centro de Interés Turístico Nacional a Aguadulce, cuyo Consejo de Administración estaba formado, entre otros, por el general Máximo Cuervo Radigales como Presidente y el propio Fernando Cassinello como Secretario General (17). Sin duda, esta circunstancia le permitió construir varios edificios tanto en Aguadulce como en la Urbanización de Roquetas de Mar -también señalada después como CITN-.

La primera obra de la que tenemos constancia se construyó en 1955 y fue un trabajo de interiorismo fruto de un encargo de su familia política, la tienda Calzados Plaza situada en la céntrica plaza almeriense de Puerta Purchena, y actualmente desaparecida (6). En 1956 construyó una vivienda aislada a las afueras de la capital almeriense denominada «La Roca» (18), acrónimo de la unión de los apellidos de Fernando Roda y María Cassinello, cuñado y hermana del arquitecto. En las décadas de los sesenta y setenta realizó muchas viviendas unifamiliares: algunas en Madrid, en la provincia de Almería y en las vecinas urbanizaciones de Aguadulce y Roquetas de Mar entre las que merece destacarse su propia casa «El Cortijillo» (1971), frente al hotel Portomagno en Aguadulce, una casa en San José, Carboneras, y un chalet de ladrillo en la urbanización madrileña del Conde Orgaz. También en el municipio de Almería, en la carretera hacia Málaga, desarrolló la Urbanización Castell del Rey y las correspondientes viviendas (Figura 4).

El Instituto Nacional de la Vivienda convocó en 1956 el Concurso de Viviendas Experimentales ${ }^{13}$ en Madrid en el que Fernando Cassinello participó con la empresa Colomina y Serrano, quedando clasificados en $5 .^{\circ}$ lugar en el apartado de viviendas en bloque y $3 .^{\circ}$ en el de adosadas. La construcción se planteó con muros de carga perpendiculares a fachada dispuestos cada $3 \mathrm{~m}$ y cerramientos exteriores con el ladrillo tipo «ceja» patentado por Miguel Fisac. La fachada estaba resuelta con un interesante juego de llenos y vacíos dispuestos al tresbolillo, proponiendo dos distribuciones diferentes para conseguir ese efecto plástico (19), (20).

Su producción arquitectónica está básicamente centrada en viviendas, aunque también construyó equipamientos, algunos de ellos vinculados con el turismo y con la emergente industria cinematográfica almeriense que requería establecimientos hoteleros para el personal de rodaje y las estrellas del momento. Destaca el Gran Hotel Almería (1967) (Figura 5), junto a la Rambla y con una posición privilegiada frente al mar y el Alcazaba Gran Hotel (1968) (21) (Figura 6), en la playa del Zapillo que pasó a ser residencia de ancianos y demolida definitivamente en 2007, además de otro hotel en San Miguel de Cabo de Gata o las cuevas del Mesón Gitano a los pies de la Alcazaba de Almería.

${ }^{10}$ Hoy se denomina International Council for Research and Innovation in Building and Construction, pero se le conocía también como International Council for Building o Consejo Internacional de la Construcción.

${ }^{11}$ A finales de 1959 impartió sendas conferencias con Eduardo Torroja en Madrid y Barcelona. Y en 1967 una ponencia titulada: «La estética del Pretensado» que impartió tanto en el IETcc, como en el Colegio de Arquitectos de Barcelona. También pronunció charlas para el $F o-$ mento de las Artes abarcando títulos tan diversos como «Ciudades de ayer, hoy y mañana», «Deformaciones ópticas», «La decoración» o «Línea y superficie» entre otros.

${ }^{12}$ Sorprende que impartiera la ponencia: «Industrialización: sistema Costamagna» el 12 de noviembre de 1968 en Sevilla y al día siguiente en Zaragoza.

${ }^{13}$ Fruto de este concurso también construyeron los arquitectos Sáenz de Oíza, Fisac, Cubillo, Carlos de Miguel, Sobrini, Álvarez Castelao, etc. 

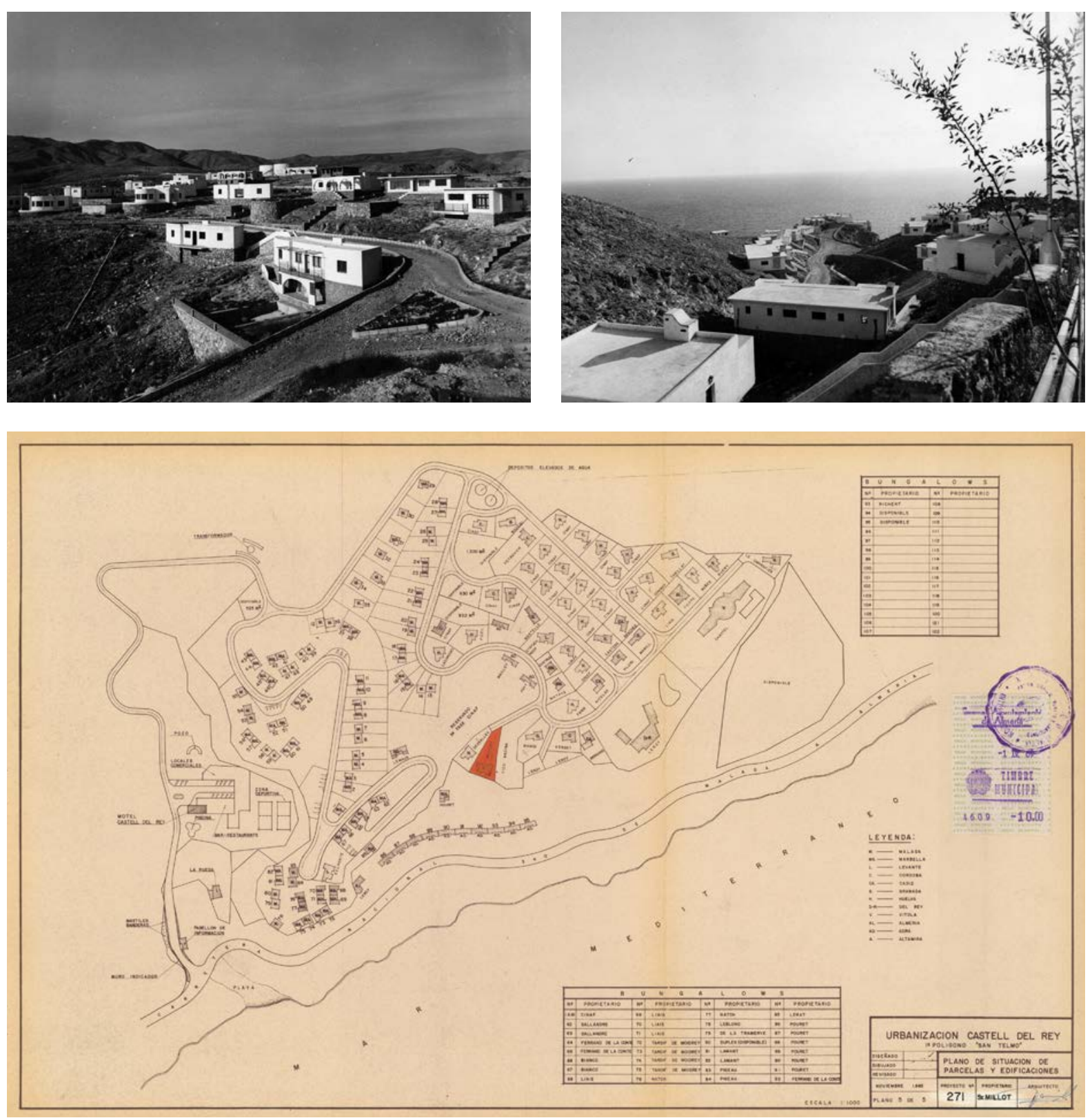

Figura 4. Fotografías y plano de la Urbanización Castell del Rey (1965)

[Fuente: Archivo del Ministerio de Información y Turismo, AGA y Archivo del Ayuntamiento de Almería].
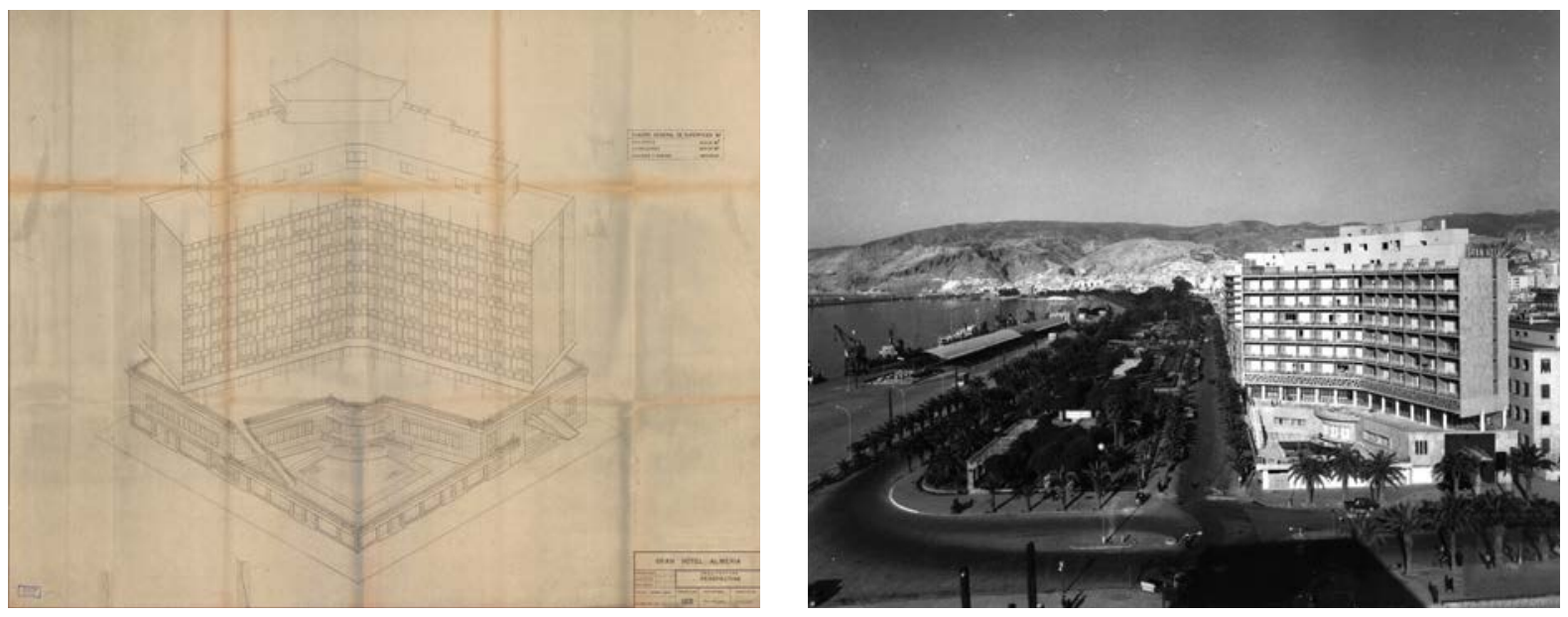

Figura 5. Perspectiva e imagen del Gran Hotel

[Fuente: Archivo de la Diputación Provincial de Almería y Archivo del Ayuntamiento de Almería]. 

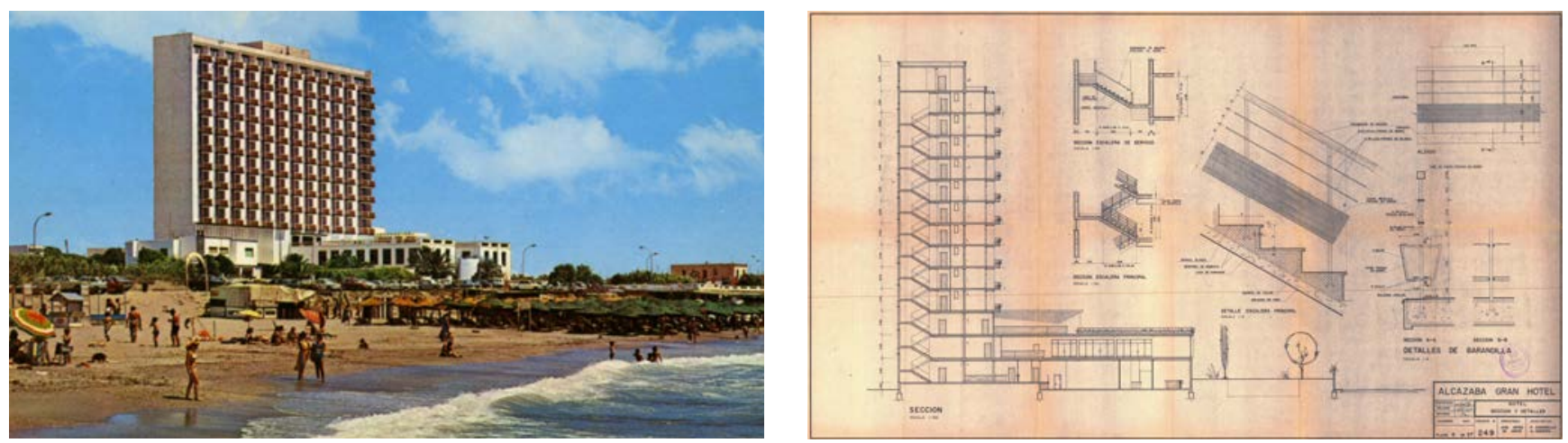

Figura 6. Imagen y sección del Hotel Alcazaba de Almería

[Fuente: Archivo de la Diputación Provincial de Almería y Archivo del Ayuntamiento de Almería].

Debe destacarse también la Capilla de Nuestra Señora de los Vientos (1972) en la Urbanización de Roquetas de Mar o la decoración interior de la capilla de Costillares (1957) en el IETcc de Madrid. En el número 214 de la revista Informes de la Construcción, publicado en 1969, se incluye un artículo titulado «Morfogénesis de una lámina» donde se explica el proyecto y construcción, en el IETcc, de una lámina de hormigón armado, tomando como base formal el elemento más característico del Centro: la costilla. Se trata de un híbrido entre un monumento estructural y una cubierta de un espacio arquitectónico para la celebración de actos diversos, también religiosos. Fernando Cassinello desarrolla la parte arquitectónica, y José Antonio Torroja, la estructural.

En relación con el Patrimonio almeriense, en 1973 redactó el «Proyecto de remodelación de la Catedral de Almería» continuando el proyecto de Francisco Prieto-Moreno desarrollado en varias etapas. En una de ellas se adecuaba el espacio bajo la parroquia del sagrario para albergar el Museo Diocesano. La obras se iniciaron a principios de 1974 y ralentizadas por cuestiones económicas se paralizaron en junio de 1975, poco antes de su fallecimiento.

En Almería capital trabajó para varios promotores construyendo centenares de viviendas, llegando a construir barrios. Para Juan Terriza Jiménez proyectó parte del barrio de San Luis (1964), en el que destaca la repetición de 15 veces de un bloque de cinco plantas formado por dos cuerpos simétricos con la escalera en uno de los extremos y acceso a las tres viviendas por corredor. Para INURNISA construyó las torres (1964) de San Félix en la barriada del mismo nombre, junto a la antigua carretera de Granada.

En 1970 realizó el pabellón de Almería para la VIII Feria Internacional del Campo. Según indica la memoria del proyecto: «Paramentos encalados; mármoles almerienses; azulejos, baldosas y celosías metálicas; rejas trenzadas a forja; plantas tropicales; tejidos de esparto y jarapas...» (22) es evidente el interés del arquitecto en aportar al edificio todos aquellos elementos que consideraba característicos de la arquitectura almeriense. La organización de la Feria premió a la Cámara Oficial Sindical Agraria de Almería, promotora del edificio, con el Diploma y Medalla de Oro «atendiendo los méritos del nuevo Pabellón principalmente» ${ }^{14}$.
Junto al arquitecto Ramón Urmeneta Ajarnaute (19181998) proyectó y construyó unas viviendas en el barrio de Orvina (1968) en Pamplona, utilizando un por entonces novedoso sistema de grandes paneles prefabricados (Figura 7).

\subsection{Paseo marítimo de Almería}

Además de los dos hoteles antes señalados, en el frente marítimo almeriense también podemos encontrar varias obras de Cassinello. Desde el centro de la ciudad hacia el Cabo de Gata se sitúan:

a) Las Caracolas (1966) es un bloque de apartamentos de 9 niveles de altura, donde destaca la disposición de huecos aleatorios en dos de los alzados, y unos elementos ligeros en las terrazas a modo de celosía.

b) El edificio Playa (1963) (Figura 8) conocido porque albergaba el restaurante Manolo Manzanilla. Además del programa de viviendas disponía de una planta de semisótano, pero abierta a la playa, para las habituales casetas de baños de la época. En las plantas superiores se disponen 12 viviendas, a razón de dos por planta, con una distribución orientada a buscar las vistas al mar. Las terrazas de las viviendas se apoyan sobre dos vigas en ménsula de sección variable, al igual que las jácenas del local, mediante voladizos de casi cuatro metros. Los cinco pórticos estructurales se convierten en la planta baja del restaurante en una singular geometría en forma de «W» de tal forma que recibe tres pilares que transforma en sólo dos apoyos. La escalera se sitúa en la parte posterior, vinculada a la galería de acceso a las viviendas, para así liberar la fachada y que ésta se vuelque plenamente hacia las vistas al Mar Mediterráneo.

c) Los Tritones (1967) es un bloque rectangular de 50 apartamentos distribuidos en 10 niveles, una planta baja donde se albergaba una cafetería-bar y una planta de semisótano de casetas de playa. El alzado que mira al mar está formado por cuatro marcos por planta, que actúan a modo de terrazas y que configuran la imagen del edificio. Destaca la forma de resolver la geometría no ortogonal del solar mediante pequeños retranqueos en cada pórtico de la estructura. Existen dos versiones del proyecto: la primera donde este último gesto no se produce $\mathrm{y}$, además,

${ }_{14}$ Medalla de Oro para el Pabellón de Almería en la Feria del Campo. Cámara Oficial Sindical Agraria de Almería. Documento del Archivo Histórico Provincial de Almería (sin fecha). 

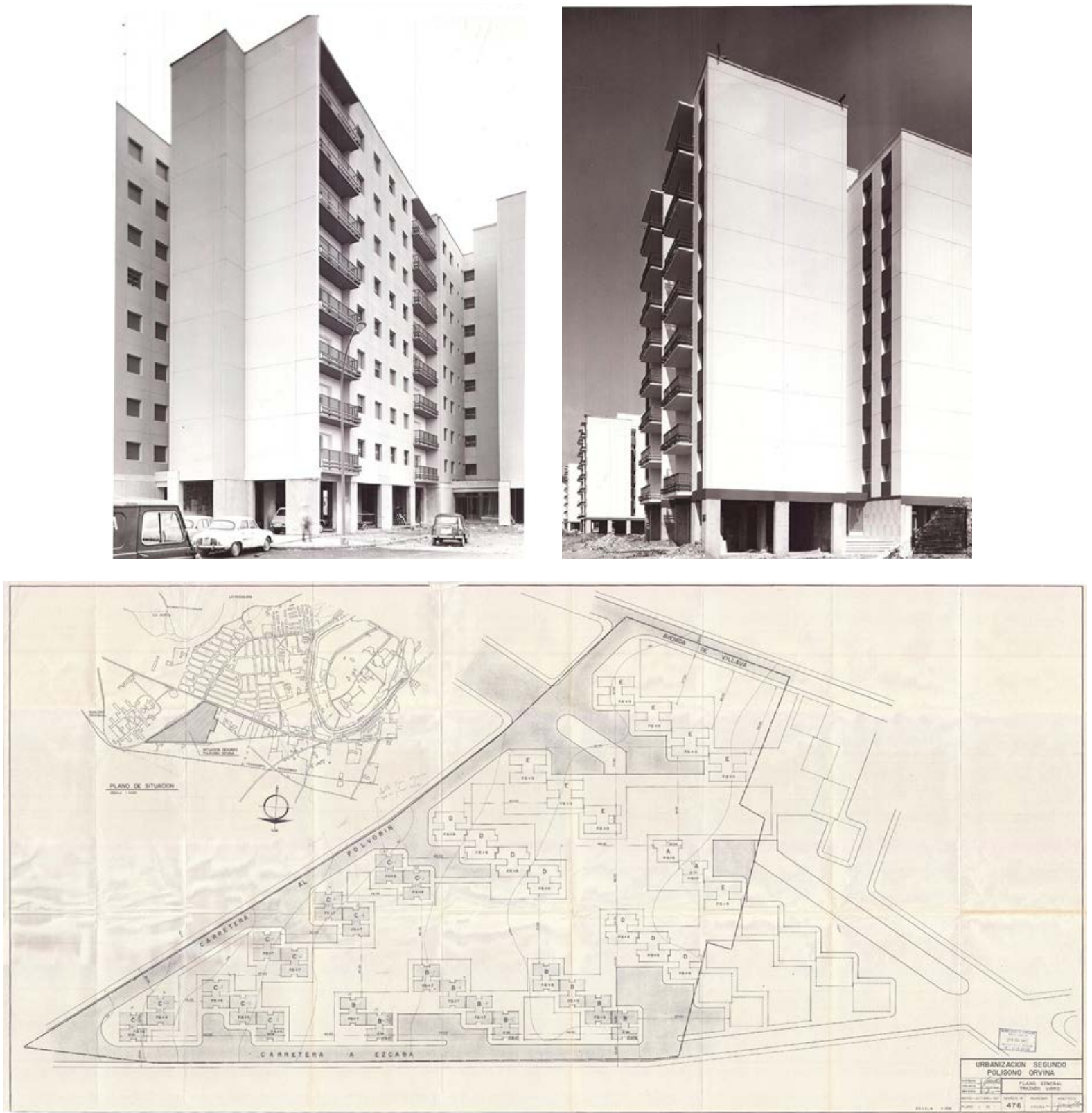

Figura 7. Fotografías y Plano de ordenación de la Urbanización Orvina en Pamplona (junto al arquitecto Ramón Urmeneta) [Fuente: Archivo del Ayuntamiento de Pamplona].

se proyectaba con un apartamento menos por planta. La segunda versión también incorporaba unos maceteros de cerámica que el autor definía como «materiales y motivos típicos almerienses» (23).

d) Las Sirenas (1965) se sitúa en segunda línea de playa, pero su altura le permitía disfrutar también de las vistas al mar desde algunos de sus 53 apartamentos. Ocupaba la esquina de la manzana y su valor residía en el aplacado cerámico de los dos alzados.

e) El Playmar (1967) constituye la aportación menos interesante debido a su densidad, y, sin embargo, veranear allí se convirtió en un símbolo de relevancia social para la incipiente clase media almeriense de los años setenta.
Se trataba de un ambicioso proyecto de tres bloques en primera línea, y cuatro más retrasados ${ }^{15}$, además de la ampliación de la playa, e incluso la construcción de unos espigones para el amarre de embarcaciones deportivas. Finalmente sólo se construyeron los tres bloques adosados más próximos al mar.

f) Las Tres Torres del «Star building» (1975) (24) se trata de uno de sus mejores obras. Su singularidad reside en que las plantas pares están giradas $45^{\circ}$ respecto a las impares. Además de la habilidad para resaltar la esbeltez debido a la pequeñez de la planta, con sólo 2 apartamentos por nivel. Se trata de la última obra de Cassinello, y su muerte coincide con la finalización de la obra, el 19 de agosto de 1975.

\footnotetext{
${ }^{15}$ En una entrevista en el periódico La Voz de Almería se le preguntaba por los problemas de circulación y por la proximidad de la edificación a la playa y el autor respondía que el proyecto había sido aprobado por el Ministerio de Obras Públicas y la Dirección General de Puertos.
}

«Almería se asoma al mar. Entrevista con el Dr. Arquitecto Don Fernando Cassinello». (3o de marzo de 1967). La Voz de Almería, p. 7. 

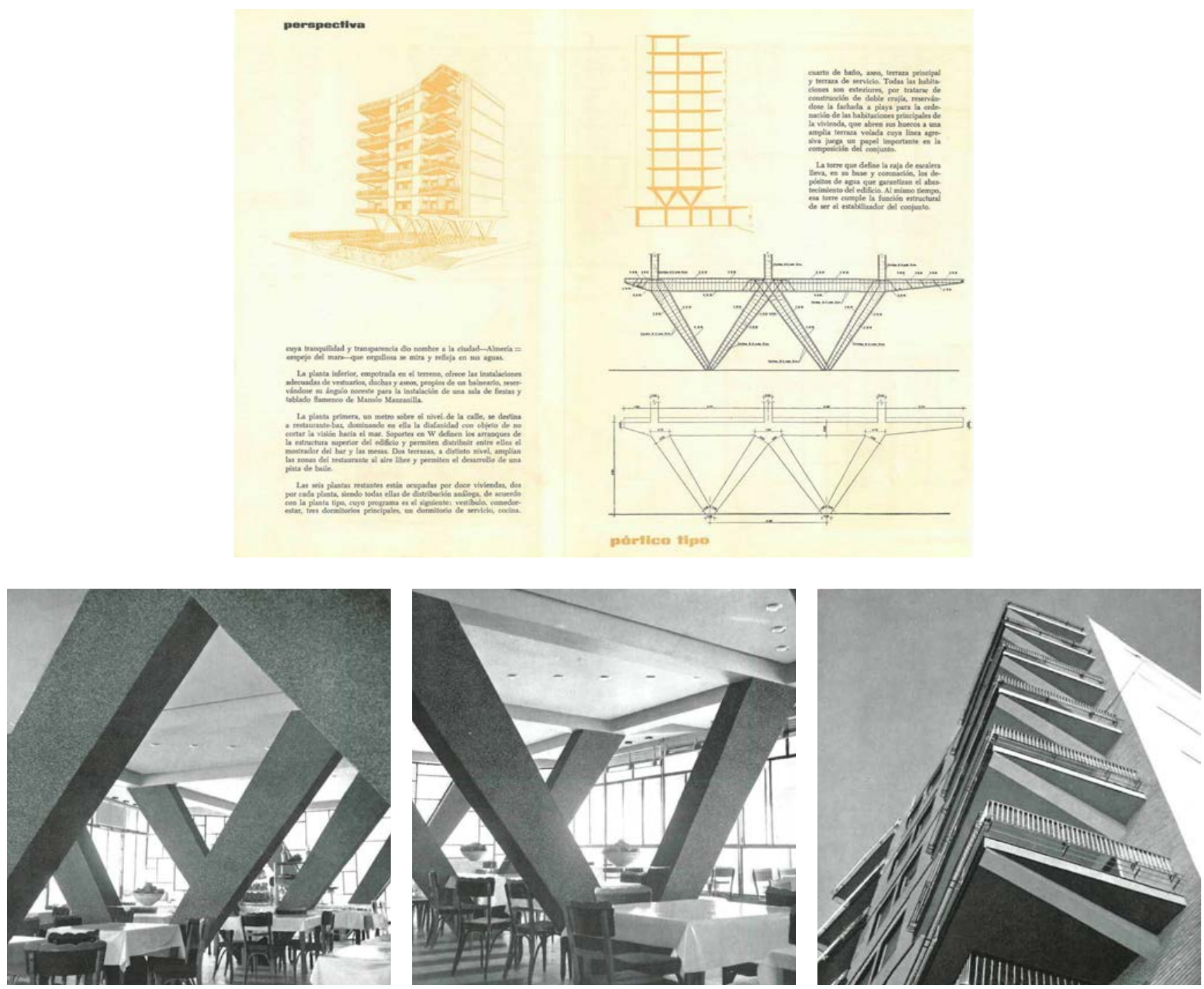

Figura 8. Planos y fotografías del Edificio Playa (1963) [Fuente: Informes de la Construcción].

\subsection{Urbanización de Aguadulce}

a) El Palmeral (1968) (25) (Figura 9), se trata de su obra más conocida. Están formados por dos bloques iguales unidos por uno de los testeros. Ambos cuentan con cuatro niveles de apartamentos -4 por planta-, quedando el conjunto total formado por 32 viviendas; además de una planta baja diáfana que la normativa así exigía. Su singularidad reside en que cada bloque de apartamentos descansa sobre tres arcos de hormigón armado, superponiendo la estructura de pórticos de los apartamentos sobre los tres grandes arcos dispuestos paralelos a la fachada. A los apartamentos se accede por la parte posterior de cada bloque mediante una escalera situada en el centro y que desemboca en una serie de galeríascorredor abiertas y que dan acceso a cada apartamento ${ }^{16}$. b) Las Yuccas (1970). Situado junto a El Palmeral, está formado por tres bloques adosados de 16 apartamentos cada uno. Además, comparte con su vecino un esquema similar de distribución en planta y las escaleras, sustituyendo los grandes arcos rebajados del primero por pórticos convencionales.

c) El Pitaco (1971). Situado a continuación de Las Yuccas, se trata de un bloque de cuatro plantas de ocho apartamentos cada una. Destaca una escalera similar a la de los edificios anteriores, pero más espectacular ya que no arranca desde el suelo, sino que comienza en una planta de acceso al edificio que la propia topografía de la parcela eleva a un nivel superior. Lamentablemente hoy esa escalera queda deslucida por la instalación de un ascensor.

\footnotetext{
${ }^{16}$ Existen dos tipos de apartamentos: de uno o dos dormitorios según sean los centrales o los de los extremos de cada bloque. En todas las viviendas, el estar-comedor mira a la fachada principal, quedando la cocina abierta a aquél. Las terrazas del estar-comedor, que vuelan sobre el plano de la fachada y de la línea estructural que marca el arco de hormigón, tienen una disposición en planta semejante a un diente de sierra, generando terrazas con forma de triángulo rectángulo que se protegen de la contigua mediante celosías cerámicas. La escalera de acceso posterior, aparentemente, es de zanca y meseta volada en su sentido longitudinal. Sin embargo, al disponer de una zanca escalonada de espesor constante de $10 \mathrm{~cm}$, requiere de una viga situada en el mismo centro de la escalera que une ambos forjados y cuyo trazado discurre paralelo al ángulo que forma la zanca; esta viga es la responsable de absorber los esfuerzos mayores.
} 

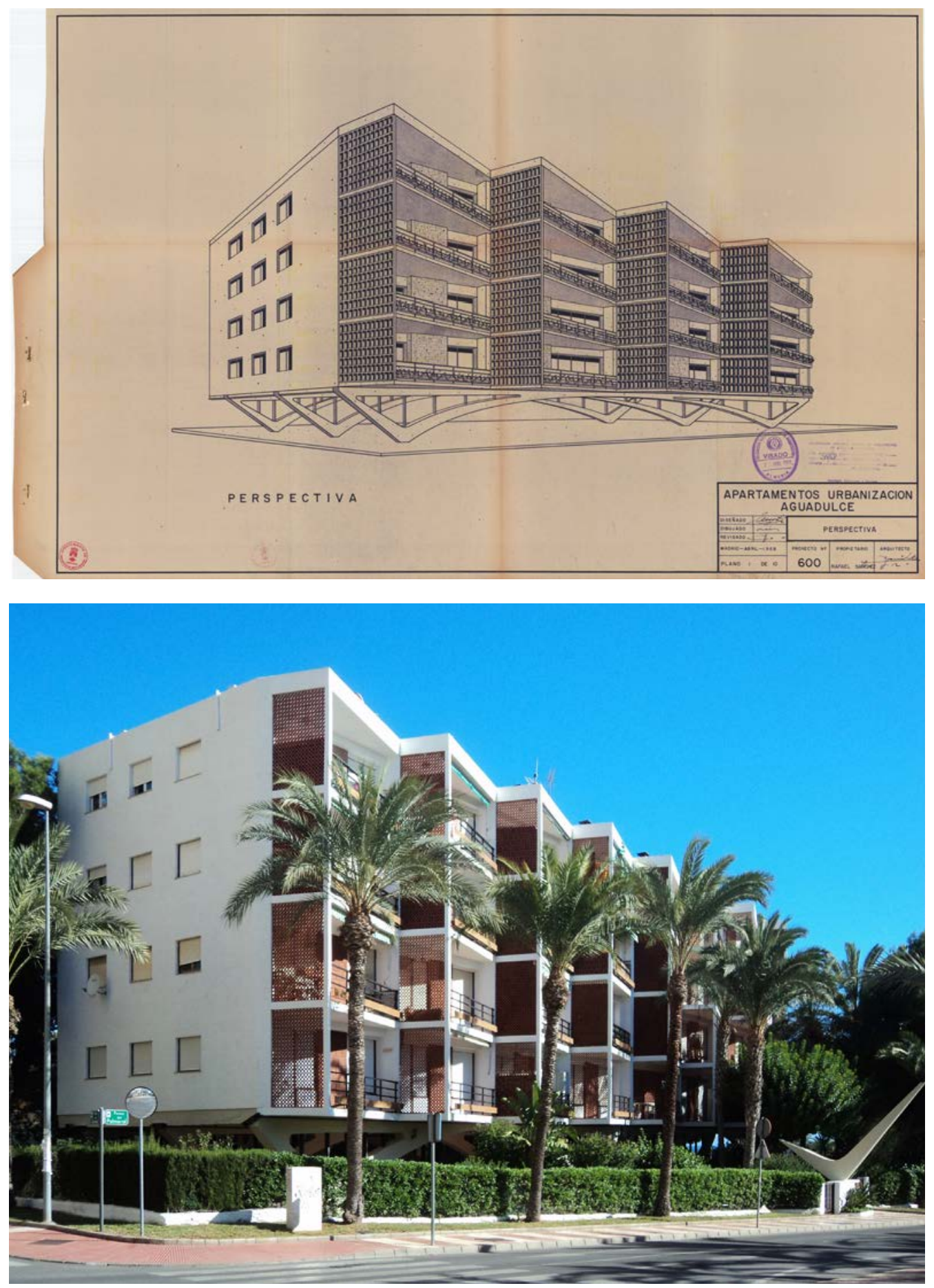

Figura 9. Edificio El Palmeral: perspectiva y estado actual.

[Fuente: Archivo del Colegio de Arquitectos de Almería y fotografía de los autores].

\subsection{Urbanización de Roquetas de Mar}

A finales de los años sesenta se desarrolla la Urbanización de Roquetas de Mar. Cassinello ya tiene notable experiencia profesional y recibe los encargos de construir casi la mayoría de los edificios de la arteria principal, la avenida del Mediterráneo: un eje perpendicular al mar. En la acera de levante:

a) Las Olas (1972). Se trata de un bloque de 32 apartamentos -8 por planta - de un solo dormitorio, que proyecta junto a Jesús Martínez Durbán, compuesto por dos volúmenes adosados, uno retranqueado respecto al otro. La solución habitual realizada por Cassinello en este tipo de edificios de apartamentos se modifica al disponer la escalera paralela al pasillo. Las terrazas reciben la forma ondulada de una "ola» que da nombre al edificio, o viceversa. Los descansillos de las escaleras, así como otros elementos, recogen esa geometría circular. Se trata del único caso de vivienda colectiva donde Cassinello traza arcos.

b) Las Gaviotas (1971) (Figura 10). Está situado junto a Las Olas y está formado por 80 apartamentos, también de un solo dormitorio. La planta tiene forma de «L», en cuyo lado mayor - paralelo a la avenida principal- se 

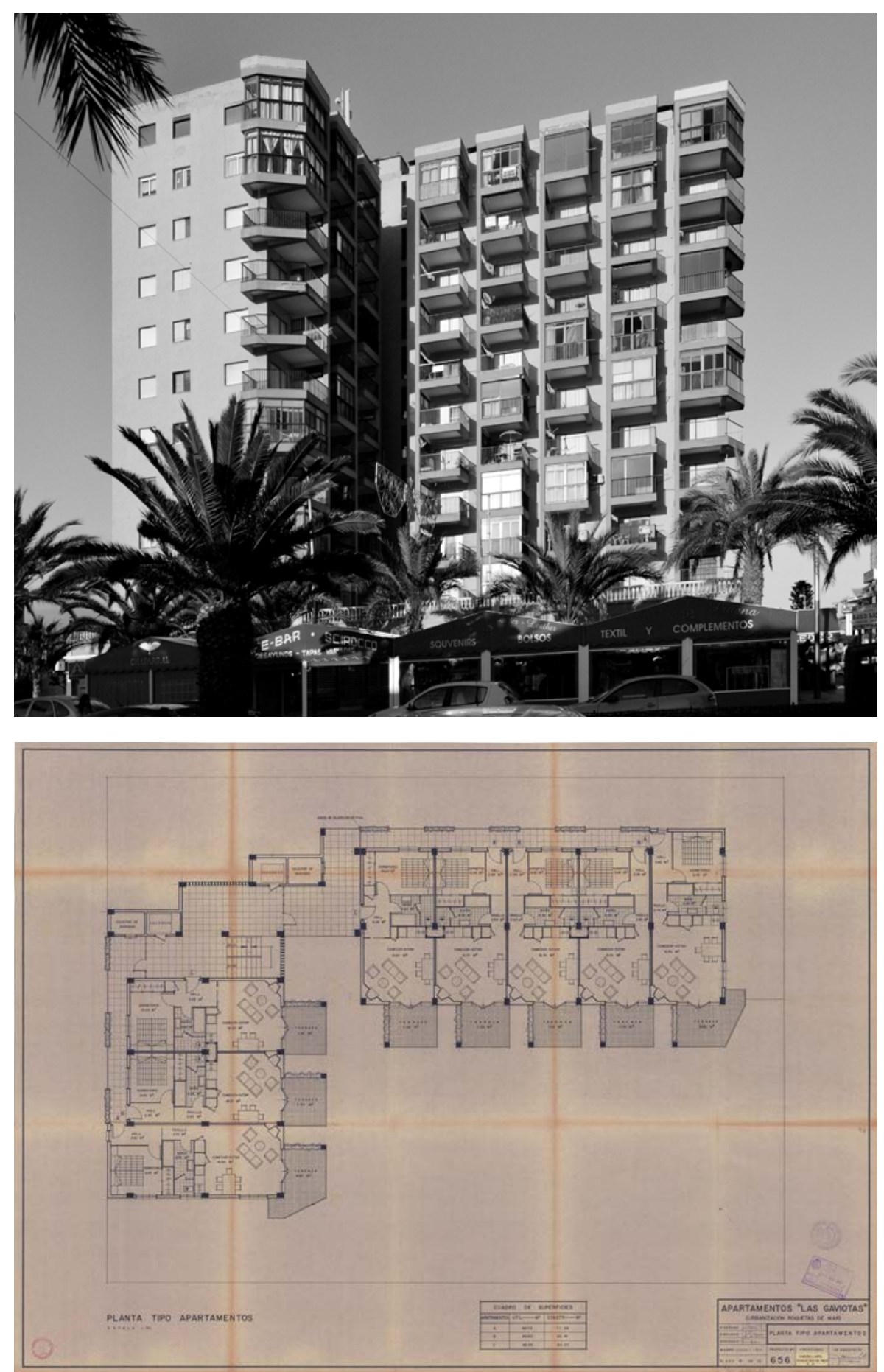

Figura 10. Edificio Las Gaviotas, estado actual.

[Fuente: Archivo del Ayuntamiento de Roquetas de Mar y fotografía de los autores].

sitúan cinco viviendas, y en el menor, tres. En el vértice se dispone el sistema de comunicaciones vertical, con una inteligente articulación que permite desde los descansillos ver hacia el exterior y a la piscina que se sitúa sobre una planta baja que ocupa toda la parcela y actúa a modo de basamento. Las terrazas, de generosas dimensiones, vuelan sobre los dos alzados que miran a la calle principal y la piscina, y se apoyan en un soporte, situado siempre en la parte interior, manifestando la voluntad de resaltar el vuelo de las terrazas.

c) Las Chumberas (1970) (Figura 11). Se trata de un conjunto formado por cuatro bloques de 20 apartamentos cada uno. Es un tipo semejante a El Palmeral, pero con una vivienda más por planta. El alzado principal lo forman unos soportales, ligados al comercio de la avenida principal; así, las terrazas de la primera planta se prolongan sobre ellos, siendo mayores que las de los niveles superiores. A diferencia de los edificios de Aguadulce, la meseta de la escalera se cierra con una celosía cerámica.

d) El Acuario - El Concordia- (1971) (Figura 12). Está formado por 80 apartamentos, sobre 10 niveles, y la planta baja dedicada a comercio. Se trata de un bloque formado por dos volúmenes retranqueados uno respecto al 

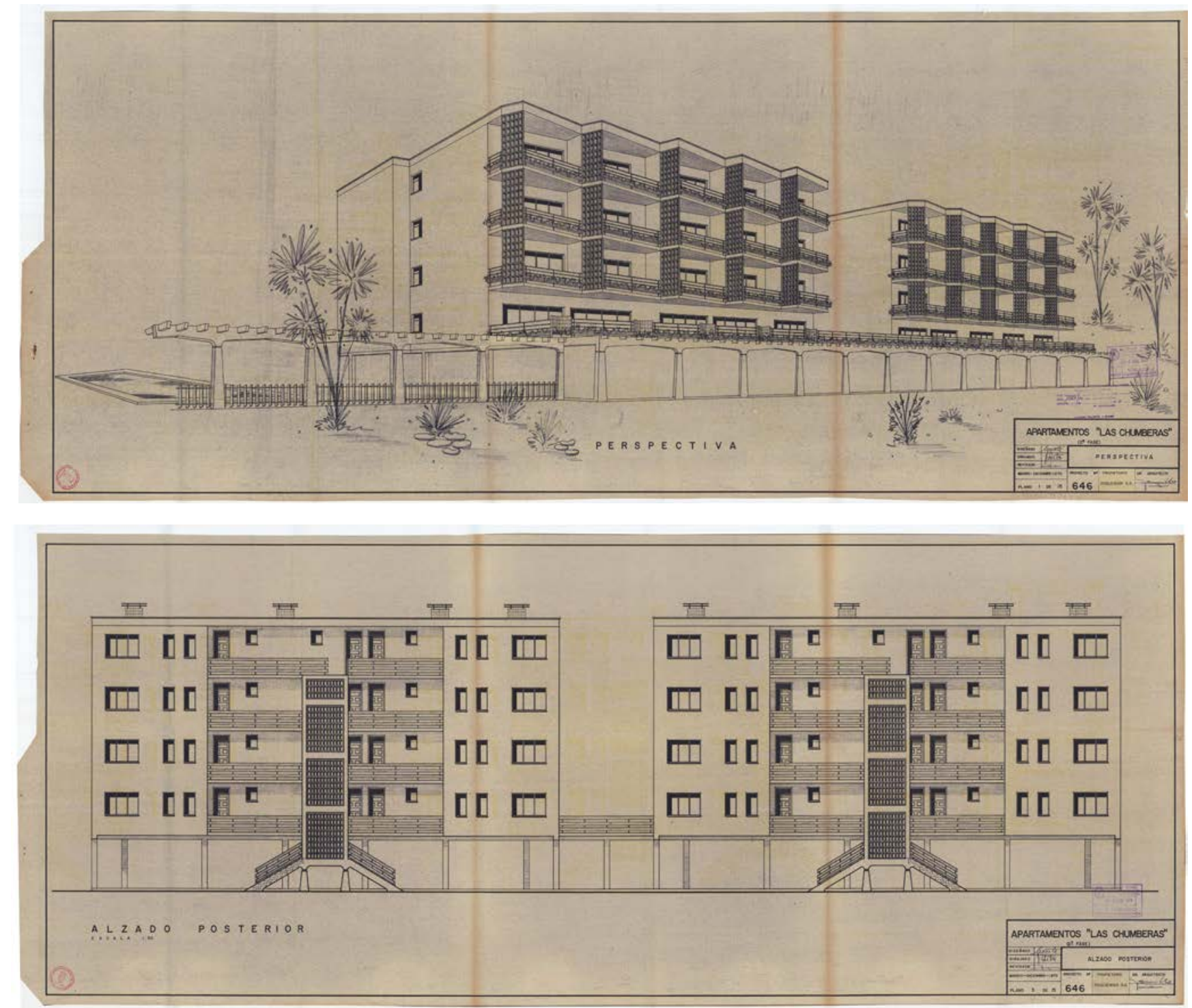

Figura 11. Perspectiva y alzado posterior (escaleras) de Las Chumberas (1970) en la Urbanización de Roquetas de Mar. [Fuente: Archivo del Ayuntamiento de Roquetas de Mar].

otro. En el más próximo a la calle, se sitúan los apartamentos de dos dormitorios, y en el otro, los apartamentos de uno ${ }^{17}$.

e) Los Géminis (1971). Está formado por dos bloques aislados, uno de ellos paralelo a la calle principal, de 6 apartamentos de un dormitorio por planta, sobre los 4 niveles de cada bloque, además de una planta baja, aquí libre. Es un tipo que recibe la forma de las terrazas de su vecino El Acuario.

f) Los Arenales (1969). Es un conjunto residencial estival formado por tres bloques orientados a levante. Su disposición en planta forma un tridente, cobijando en el centro la piscina y las zonas de esparcimiento. El bloque central, de 10 niveles de altura y 45 viviendas, preside el conjunto girado $45^{\circ}$ respecto a la trama urbana, escoltado por dos bloques, de 4 niveles de altura y 12 viviendas cada uno, en este caso los edificios están alineados a las avenidas de las Gaviotas y Mediterráneo, respectivamente. Cada bloque cuenta con una escalera helicoidal exterior situada en el mismo centro del alzado posterior, donde los peldaños prefabricados vuelan desde un fuste central formado por un semicilindro hueco de hormigón armado. La singularidad reside en que, siguiendo los cánones marcados por los CIAM, se sitúa el centro cívico del conjunto elevado y abierto en el tercer nivel del bloque mayor, comunicándose éste, mediante pasarelas, con los dos edificios pequeños laterales (25).

g) Los Jopos (1973) (Figura 13). Se trata de dos bloques de 16 apartamentos cada uno, situados en una generosa parcela, muy cerca de Los Arenales. La escalera, también de geo-

\footnotetext{
${ }^{17}$ A cada vivienda se accede desde un sistema de galerías cerradas con una interesante celosía formada por piezas hexagonales. Esta geometría se repite, también en la planta de la escalera, en la piscina, o en la forma de las terrazas, si bien en este último caso, su trazado es de un hexágono irregular. Éstas se apoyan sobre jácenas que vuelan sobre la calle y cuya superficie puede considerarse más que generosa: desde los $16,60 \mathrm{~m}^{2}$ de la terraza en esquina de los apartamentos de dos dormitorios, hasta los $10 \mathrm{~m}^{2}$ de los que disponen de un dormitorio. Las terrazas ordenan el alzado y, junto con el sistema de celosías, se convierten en la imagen de esta obra.
} 

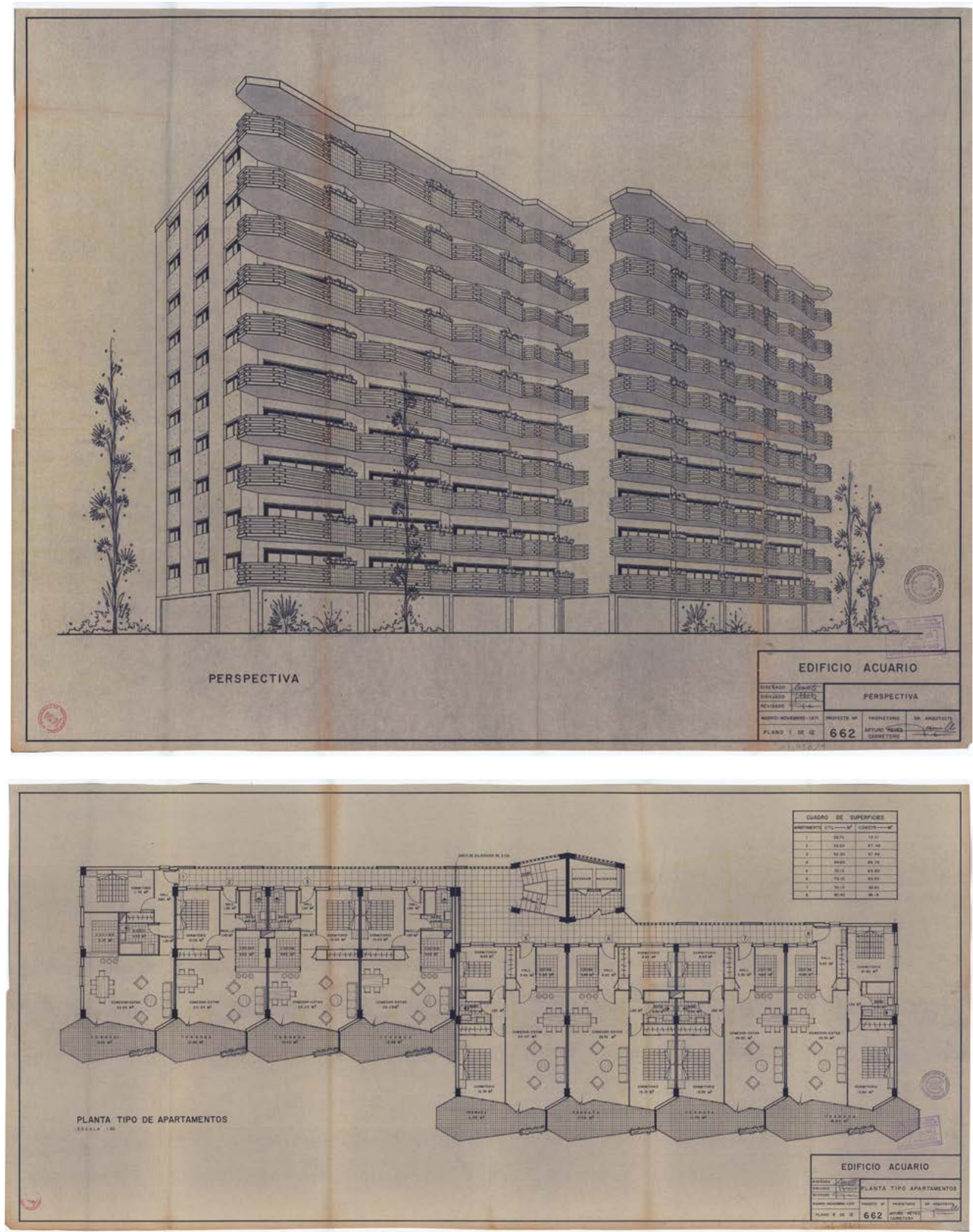

Figura 12. Perspectiva y planta de El Concordia (Acuario) (1971), Urbanización Roquetas de Mar.

[Fuente: Archivo del Ayuntamiento de Roquetas de Mar].

metría helicoidal, se sitúa en la parte posterior y desemboca en unos corredores abiertos por los que se accede a cada apartamento. La diferencia, respecto a Los Arenales, reside en que aquí la escalera está cerrada por un muro de ladrillo, construido mediante fábrica premeditadamente imperfecta - produciendo 'rebabas' en las juntas que presentan una interesante textura al incidir la luz-. La caja de escalera semicircular está perforada por huecos de formato rectangular y disposición aleatoria, albergando una pieza de 'pavés' cada uno cuyos colores pigmentan la luz. 

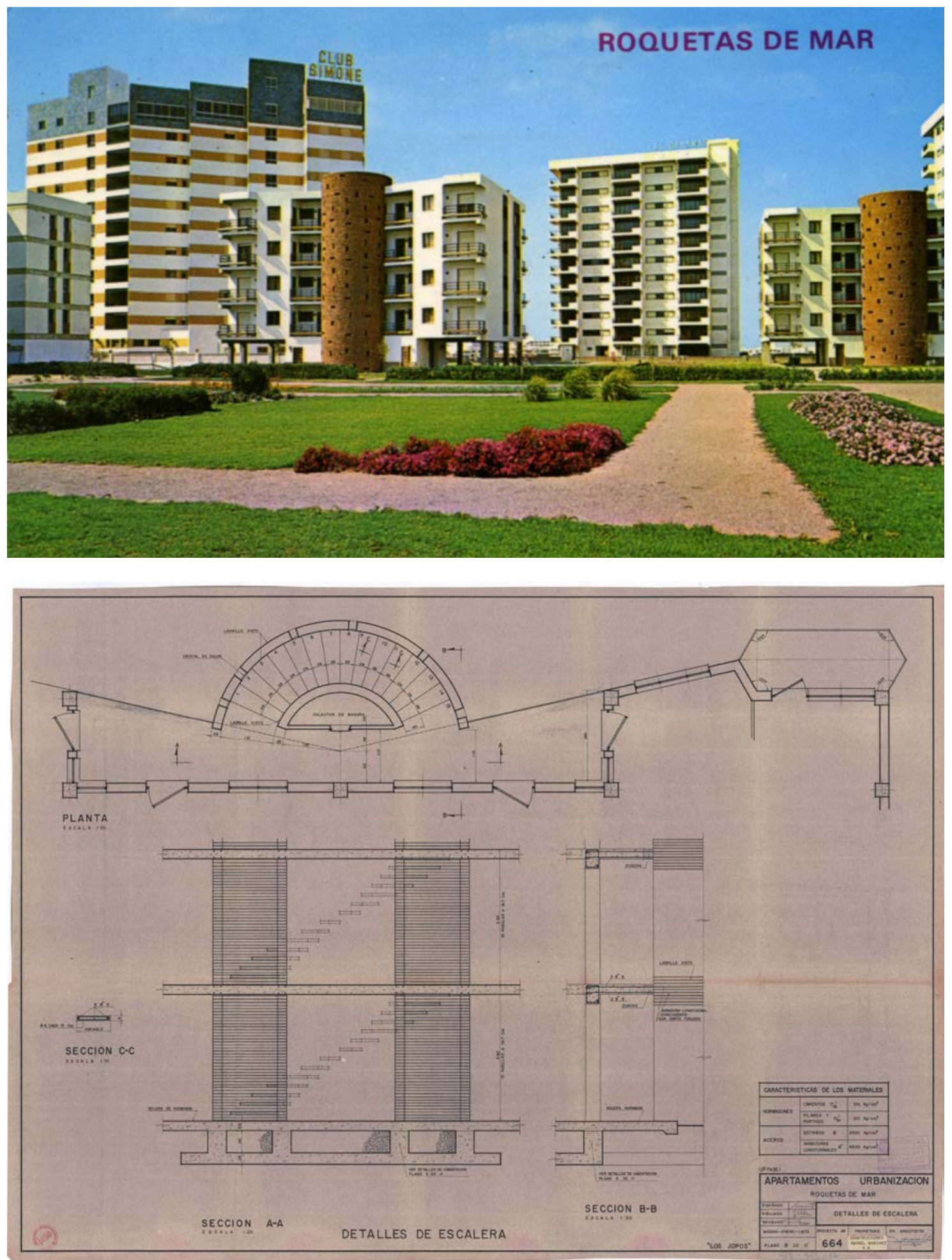

Figura 13. Detalle de la escalera helicoidal de Los Jopos (1972), Urbanización de Roquetas de Mar, y postal de la época donde se aprecian las cajas de escaleras construidas con piezas cerámicas.

[Fuente: Archivo del Ayuntamiento de Roquetas de Mar y Archivo de la Diputación de Almería].

\section{CONCLUSIÓN}

La trayectoria de Fernando Cassinello se desarrolló de forma meteórica en cualquiera de las tres facetas descritas: la profesional, la docente y la investigadora. En este sentido forma parte de esa generación de brillantes arquitectos que compaginaron la vida en las aulas como docente con la construcción de hermosas obras, haciendo de la transferencia tecnológica del conocimiento algo que se producía de forma natural. Tuvo la suerte de coincidir con los mejores: Sáenz de Oíza, Fisac, Torroja; además de relacionarse con figuras internacionales como Frank Lloyd Wright, Richard Neutra o el mismísimo Le Corbusier, a quien entrevistó personalmente.

Su arquitectura se sitúa en la segunda generación del movimiento moderno, es decir, la que compaginaba la voluntad de 
pertenecer a un lenguaje universal -y que el uso del hormigón favorecía-, pero también la que dialoga con las peculiaridades del paisaje, de la atmósfera cultural y del clima donde le ha tocado establecerse. Una de las aportaciones de Cassinello fue la de introducir aspectos estructurales en edificios de viviendas, donde El Palmeral de Aguadulce pudiera haberse convertido en un caso paradigmático a nivel mundial. También encontró en las escaleras un elemento donde depositar el ingenio creativo y un tema sobre el que escribir e investigar. A veces el interés residía en la escalera propiamente dicha; otras, en la caja contenedora, donde celosías caladas, muros de hormigón o de ladrillo convertían el movimiento vertical en un sensual paseo. Supo hacer de las escaleras un elemento del paisaje urbano, un escenario de encuentro de la colectividad, e, incluso, el símbolo reconocible de una obra de arquitectura.

Lo que en este artículo se cuenta es un resumen de la vida de Fernando Cassinello Pérez y que se truncó cuando sólo tenía 47 años. Nadie podrá saber hasta dónde hubiera llegado, pero nos lo imaginamos.

\section{REFERENCIAS}

(1) Ruiz-García, A. (1992). Arquitectura, vivienda y reconstrucción en la Almería de posguerra (1939-1959) [tesis doctoral]. Granada: Universidad de Granada.

(2) Aroca-Hernández-Ros, R. (2011). Compañeros: Fernando Cassinello. En Cajón de recortes (pp. 209-210). Madrid: Mairea Libros.

(3) Cassinello-Plaza, P., y Torroja, J. A. (2008). El legado oculto de una revista / The journal's hidden legacy. En CalaveraRuiz, J., Cassinello, P., Fernández-Alba, A., et al., El espíritu impreso de una idea (pp. 193-194). Madrid: Instituto de Ciencias de la Construcción Eduardo Torroja, CSIC.

(4) Cassinello-Pérez, F. (1952). La Iglesia de la Ascensión en Schaffhausen de Walter Henne y Capilla rural en la Alta Baviera de J. Ludwig. Informes de la Construcción, 5(43).

(5) Cassinello-Pérez, F. (1955). Arquitectura escolar. Informes de la Construcción, 7(74).

(6) Cassinello-Pérez, F. (1955). Nuevo local comercial en Almería. Informes de la Construcción, 8(75).

(7) Cassinello-Pérez, F. (1957). Capilla en Costillares. Informes de la Construcción, 9(87).

(8) Cassinello-Pérez, F. (1957). Capilla en el Instituto Técnico de la Construcción. Revista Nacional de Arquitectura, 189 : 15.

(9) Cassinello-Pérez, F. (1961). Eduardo Torroja. Cuadernos de Arquitectura, 46: 6-8.

(10) Cassinello-Pérez, F. (1971). Edificios para apartamentos (El Palmeral). Cuadernos de Arquitectura, 84: 47. Barcelona: COACB, Colegio de Arquitectos de Catalunya y Baleares.

(11) Cassinello-Pérez, F. (1970). Edificios de apartamentos (Los Tritones). Cuadernos de Arquitectura, 78: 61. Barcelona: COACB, Colegio de Arquitectos de Catalunya y Baleares.

(12) Fisac-Serna, M. (1989). Documentos de Arquitectura, 10. Almería: Colegio de Arquitectos de Almería.

(13) Torroja, J. (2014). Mi paso por «el Torroja». Informes de la Construcción, 66(536): edoo4 Consultado el o6-05-2016, en http://informesdelaconstruccion.revistas.csic.es/index.php/informesdelaconstruccion/article/view/3641/4122

(14) Cassinello-Plaza, P. (2009). La Historia de la Construcción a través de la revista Informes de la Construcción. En Huerta, S., Marín, R., et al. (Eds.), Actas del Sexto Congreso Nacional de Historia de la Construcción. Madrid: Instituto Juan de Herrera.

(15) Cassinello-Pérez, F. (1965). Le Corbusier. Informes de la Construcción, 18(174).

(16) Centellas-Soler, M., Ruiz-García, A., García-Pellicer López, P. (2009). Los pueblos de colonización en Almería. Arquitectura y desarrollo para una nueva agricultura. Almería: Colegio de Arquitectos de Almería, Instituto de Estudios Almerienses y Fundación Cajamar.

(17) Cirre-Jiménez, J. (1968, 30 de abril). Visita a Almería del Ministro de Información y Turismo Manuel Fraga. La Voz de Almería, pp. 10-11.

(18) Cassinello-Pérez, F. (1958). La Roca. Informes de la Construcción, 11(105), https://doi.org/10.3989/ic.1958.v11. i105.5476.

(19) Sambricio-Rivera, C., Díez-Medina, C., Cassinello Plaza, P., et al. (1997). La vivienda experimental. Concurso de viviendas experimentales de 1956. Madrid: Fundación Cultural COAM.

(20) Centellas-Soler, M., y García-Sánchez, J. F. (2014). Fernando Cassinello: un arquitecto por descubrir. En Congreso Nacional Pioneros de la Arquitectura Moderna Española: Vigencia de su Pensamiento y Obra (pp. 197-206). Madrid: Fundación Alejandro de la Sota y Ministerio de Fomento.

(21) Centellas-Soler, M., y García-Sánchez, J. F. (2015). Alcazaba Gran Hotel: Fernando Cassinello y la arquitectura turística. En II Congreso Nacional Pioneros de la Arquitectura Moderna Española: Aprender de una Obra (pp. 165-175). Madrid: Fundación Alejandro de la Sota y Ministerio de Fomento.

(22) Proyecto de Pabellón de Almería en la Feria del Campo de Madrid. Memoria descriptiva. Documento en el Archivo Histórico del Ayuntamiento de Almería (julio 1969).

(23) Cassinello-Pérez, F. (1970). Edificios de apartamentos (Los Tritones). Cuadernos de Arquitectura, 78: 61. Barcelona: COACB, Colegio de Arquitectos de Catalunya y Baleares.

(24) García-Sánchez, J. F. (2014). Tres Torres y un mar: La última obra de Fernando Cassinello. Informes de la Construcción, 66(536): e047, doi: http://dx.doi.org/10.3989/ic.14.013.

(25) García-Sánchez, J. F., y Centellas-Soler, M. (2014). Fernando Cassinello: la construcción del paseo vertical. En Miradas a la investigación arquitectónicas: construcción, gestión, tecnología (Architectural research findings: building construction, management, technology). Congreso Internacional sobre investigación en Construcción y Tecnología Arquitectónicas (pp. 478-483). Madrid: Escuela Técnica Superior de Arquitectura de Madrid. 